\title{
Erratum to: Yield stability and lower susceptibility to abiotic stresses of improved open-pollinated and hybrid maize cultivars
}

\author{
Marcos A. Lana ${ }^{1,2}$ - Frank Eulenstein ${ }^{2} \cdot$ Sandro L. Schlindwein ${ }^{3} \cdot$ Frieder Graef $^{2}$ • \\ Stefan Sieber ${ }^{2} \cdot$ Henrique von Hertwig Bittencourt ${ }^{4}$
}

Published online: 3 August 2017

(C) INRA and Springer-Verlag France SAS 2017

Erratum to: Agron. Sustain. Dev.

https://doi.org/10.1007/s13593-017-0442-x

Please replace Table 1 in above mentioned research article, published online 24 July 2017, with the version published in this erratum.

The online version of the original article can be found at http://dx.doi.org/ 10.1007/s13593-017-0442-x

Marcos A. Lana

marcos.lana@slu.se; lana@zalf.de

1 Department of Crop Production Ecology, Swedish University of Agricultural Sciences, Ulls väg 16, 75651 Uppsala, Sweden

2 Leibniz Centre for Agricultural Landscape Research (ZALF), Eberswalder Strasse 84, 15374 Müncheberg, Germany

3 Center of Agricultural Sciences, Federal University of Santa Catarina State (CCA/UFSC), Rod. Admar Gonzaga 1346, Florianópolis, SC 88034, Brazil

4 Universidade Federal da Fronteira Sul, Campus Laranjeiras do Sul, BR 158, Km 07, Caixa Postal 106, Laranjeiras do Sul, PR 85301-970, Brazil 
Table 1. Cultivar coefficients of four maize cultivars calculated by GLUE using field measurements (on top), followed by model validation outcomes for phenology (anthesis and physiological maturity) and yield.

\begin{tabular}{|c|c|c|c|c|c|c|c|}
\hline \multirow{6}{*}{ Cultivar coefficients } & & \multicolumn{6}{|c|}{ Model input genetic coefficients } \\
\hline & & $\mathrm{P} 1\left({ }^{\circ} \mathrm{C}\right.$ day $)$ & P2 (days) & P5 $\left({ }^{\circ} \mathrm{C}\right.$ day $)$ & G2 (number) & G3 (mg.day ${ }^{-1}$ ) & PHINT $\left({ }^{\circ} \mathrm{C}\right.$ day) \\
\hline & AS1548 & 271.7 & 1.387 & 997.1 & 502.1 & 6.590 & 46.17 \\
\hline & FORTUNA & 253.3 & 1.992 & 982.2 & 459.8 & 7.244 & 55.66 \\
\hline & IVANIR & 369.9 & 0.929 & 932.5 & 619.9 & 7.764 & 35.65 \\
\hline & MPA01 & 316.9 & 0.467 & 1009.0 & 623.5 & 6.599 & 58.33 \\
\hline & & \multicolumn{6}{|c|}{ Model validation } \\
\hline & & \multicolumn{2}{|c|}{$\begin{array}{l}\text { Root mean square } \\
\quad \text { error (RMSE) }(\%)\end{array}$} & \multicolumn{2}{|c|}{$\begin{array}{l}\text { Coefficient of residual } \\
\text { mass (CRM) }(\%)\end{array}$} & Simulated (average) & Observed (average) \\
\hline \multirow[t]{4}{*}{ Anthesis (days after planting) } & AS1548 & \multicolumn{2}{|c|}{2.9} & \multicolumn{2}{|l|}{-1.0} & 71 & 70 \\
\hline & FORTUNA & \multicolumn{2}{|l|}{2.4} & \multicolumn{2}{|l|}{-1.3} & 80 & 79 \\
\hline & IVANIR & \multicolumn{2}{|l|}{6.1} & \multicolumn{2}{|l|}{-4.1} & 76 & 73 \\
\hline & MPA01 & \multicolumn{2}{|l|}{6.1} & \multicolumn{2}{|l|}{-4.1} & 76 & 73 \\
\hline \multirow[t]{4}{*}{ Maturity (days after planting) } & AS1548 & \multicolumn{2}{|l|}{3.1} & \multicolumn{2}{|l|}{2.5} & 129 & 132 \\
\hline & FORTUNA & \multicolumn{2}{|l|}{12.9} & \multicolumn{2}{|l|}{-5.8} & 145 & 137 \\
\hline & IVANIR & \multicolumn{2}{|l|}{2.8} & \multicolumn{2}{|l|}{0.3} & 131 & 131 \\
\hline & MPA01 & \multicolumn{2}{|l|}{2.4} & \multicolumn{2}{|l|}{-2.1} & 143 & 140 \\
\hline \multirow[t]{4}{*}{ Yield $\left(\mathrm{kg} / \mathrm{ha}^{-1}\right)$} & AS1548 & \multicolumn{2}{|l|}{10.9} & \multicolumn{2}{|l|}{9.1} & 4729 & 5051 \\
\hline & FORTUNA & \multicolumn{2}{|l|}{13.1} & \multicolumn{2}{|l|}{2.4} & 5901 & 6044 \\
\hline & IVANIR & \multicolumn{2}{|l|}{39.8} & \multicolumn{2}{|l|}{-31.2} & 5892 & 4490 \\
\hline & MPA01 & \multicolumn{2}{|l|}{12.5} & \multicolumn{2}{|l|}{-7.8} & 5762 & 5347 \\
\hline
\end{tabular}

P1: thermal time from seedling emergence to the end of the juvenile phase (degree days above the base temperature of $8{ }^{\circ} \mathrm{C}$ ) during which the plant is not responsive to photoperiod.

P2: extent to which development is delayed for each hour increase in photoperiod above the longest photoperiod at which development proceeds at a maximum rate $(12.5 \mathrm{~h})$.

P5: thermal time from silking to physiological maturity (above a base temperature of $8{ }^{\circ} \mathrm{C}$ ).

G2: maximum possible number of kernels per plant.

G3: kernel filling rate during the linear grain filling stage under optimum conditions ( $\mathrm{mg} \mathrm{day}^{-1}$ ).

PHINT: phyllochron interval $=$ thermal time $($ degree days) between leaf tip appearances. 